\title{
Central Retinal Vein Occlusion Secondary to Ocular Trauma in a Young Patient: A Case Report
}

\author{
Ailia Ahmed, OD \\ National Vision
}

Justin Simbulan, OD

The University of Houston

College of Optometry

Bhagya Segu, OD, MPH, FAAO

Michael E. DeBakey VA

Medical Center

Angelina Tran, OD, FAAO Michael E. DeBakey VA

Medical Center

\begin{abstract}
Central retinal vein occlusions (CRVO) occurring in young patients or due to a traumatic etiology are uncommon. This paper outlines the case of a 33-year-old Asian male who presented with a chief complaint of blurry peripheral vision and photophobia in his left eye, one week after sustaining blunt ocular trauma. He was diagnosed with a traumatic CRVO based on history and exam findings.
\end{abstract}

KEY WORDS:

CRVO, blunt trauma

\section{INTRODUCTION}

After diabetic retinopathy, retinal vein occlusions are the second-most common cause of retinal vascular disorders. Branch retinal vein occlusions are more common, with a prevalence in the US population of $0.5 \%$ to $1.2 \%$, compared to CRVOs, which occur at a prevalence of $0.1 \%$ to $0.5 \% .{ }^{1} \mathrm{CRVO}$ typically presents as sudden-onset, unilateral painless vision loss in vasculopathic individuals who are 50 years of age or older. CRVO is rare in individuals 40 years old or younger, and for these individuals etiologies such as oral contraceptive use, collagen vascular disease, acquired immune deficiency syndrome (AIDS), protein S/protein C deficiency, anti-thrombin III deficiency, factor XII deficiency, antiphospholipid antibody syndrome or activated protein $\mathrm{C}$ resistance should be considered. ${ }^{2}$ In rare cases, a CRVO can be a clinical manifestation of ocular trauma.

\section{CASE REPORT}

\section{History}

A 33-year-old Asian male presented with a chief complaint of blurry peripheral vision and photophobia in his left eye for one week. He reported a recent history of trauma that occurred two weeks prior; he was hit in the left eye during Jiu Jitsu practice. The peripheral visual blur and photophobia occurred one week after the injury. The patient's ocular history revealed compound myopic astigmatism with a history of laser-assisted in situ keratomileusis (LASIK). The patient's medical history was unremarkable and he was not taking any medications. He had no known allergies.

\section{Clinical Exam}

The patient's best corrected visual acuity (BCVA) was 20/20 OD, OS. His pupils were equal in size, round, and reactive to light with no afferent pupillary defect (APD). Extraocular motilities were unrestricted and he demonstrated full confrontation visual fields OU. The slit lamp examination of the anterior segment was unremarkable except for trace cells OS. No ecchymosis, edema or abnormal ocular adnexa findings were noted OU. His intraocular pressures (IOP) measured by Goldmann tonometry were $10 \mathrm{mmHg}$ OD and 11 $\mathrm{mmHg}$ OS. Upon dilated fundus examination (DFE), the right eye was unremarkable and the left eye was significant for optic disc edema with peripapillary hemorrhages, diffuse parafoveal flame hemorrhages in the macula and flame hemorrhages and microaneurysms in all four peripheral quadrants of 
the retina (Figures 1,2). This was an atypical presentation of CRVO as hemorrhaging in this case was concentrated around the disc, with less severe bleeding away from the posterior pole. This may have been due to significant pressure on the optic nerve itself due to trauma, as opposed to the vasculopathic etiology of other CRVOs. Optical coherence tomography (OCT) of the macula demonstrated a flat and intact macula with no subretinal or intraretinal fluid OU (Figure 3). OCT of the retinal nerve fiber layer was within normal limits OD and was elevated 360 degrees OS, confirming peri-papillary edema (Figure 4). The patient's in-office blood pressure was $123 / 73 \mathrm{mmHg}$.

Figures 1 and 2: Right and left posterior poles with extensive hemorrhages OS
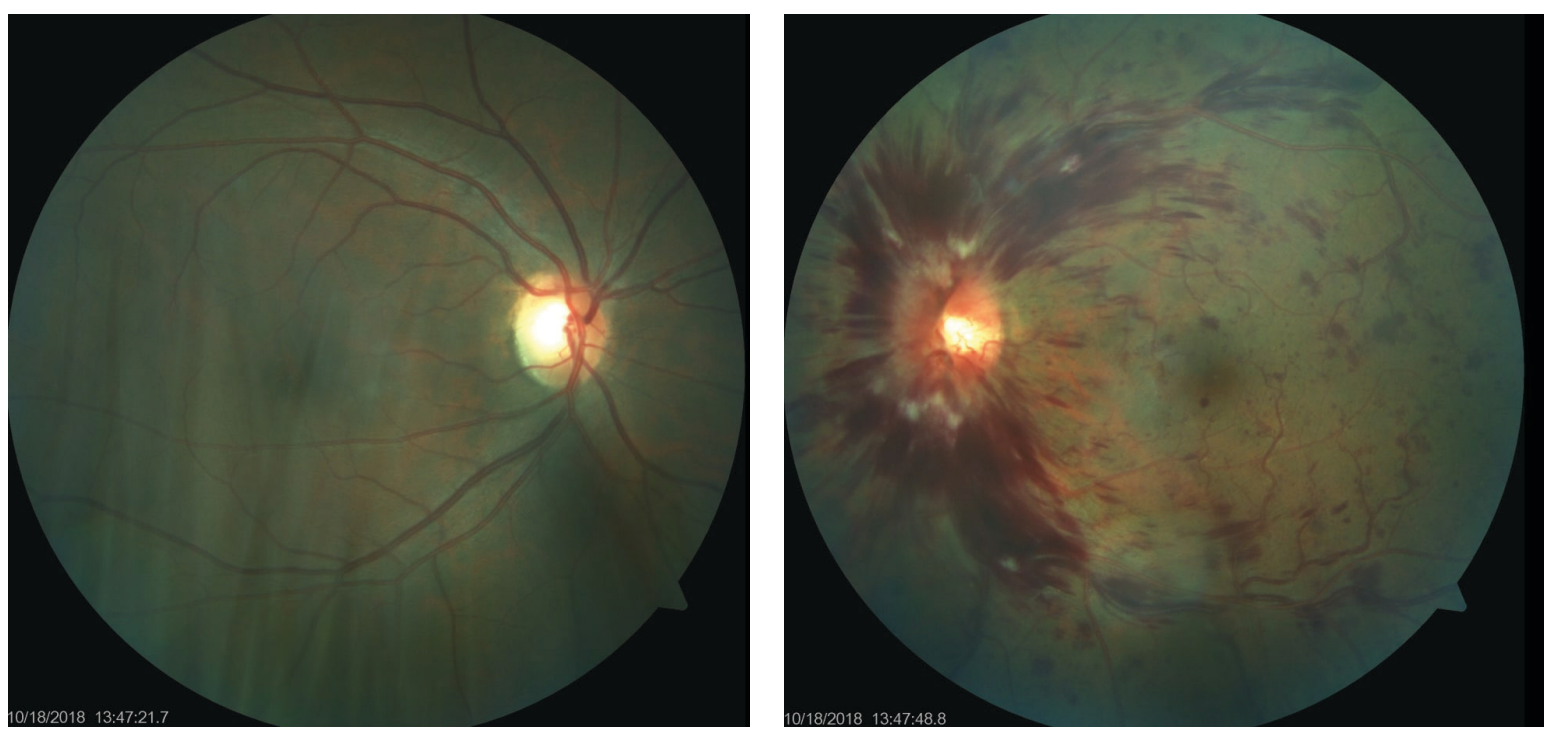

Figure 3: Macular OCTs with mild increased macular thickening OS
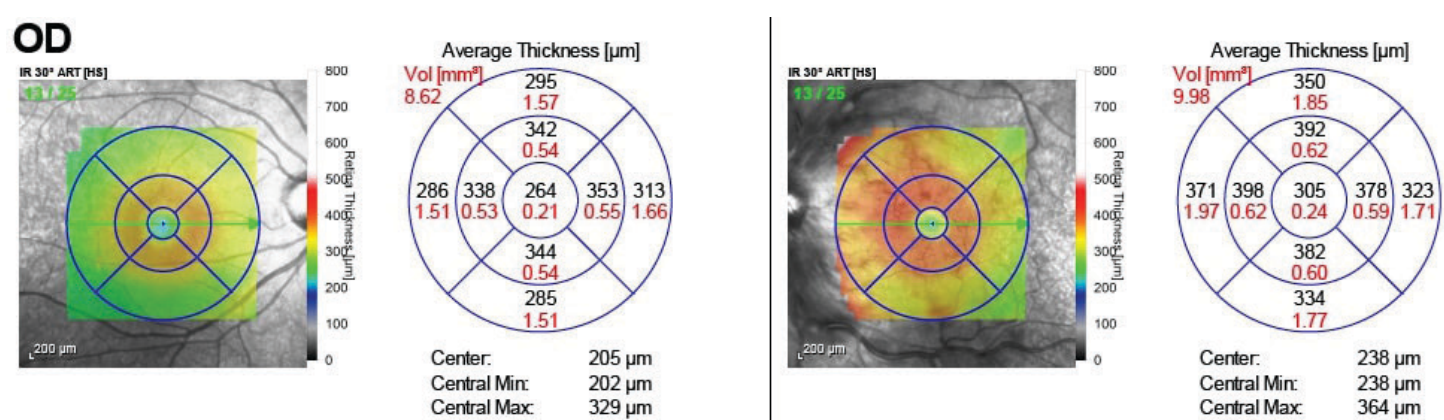

os
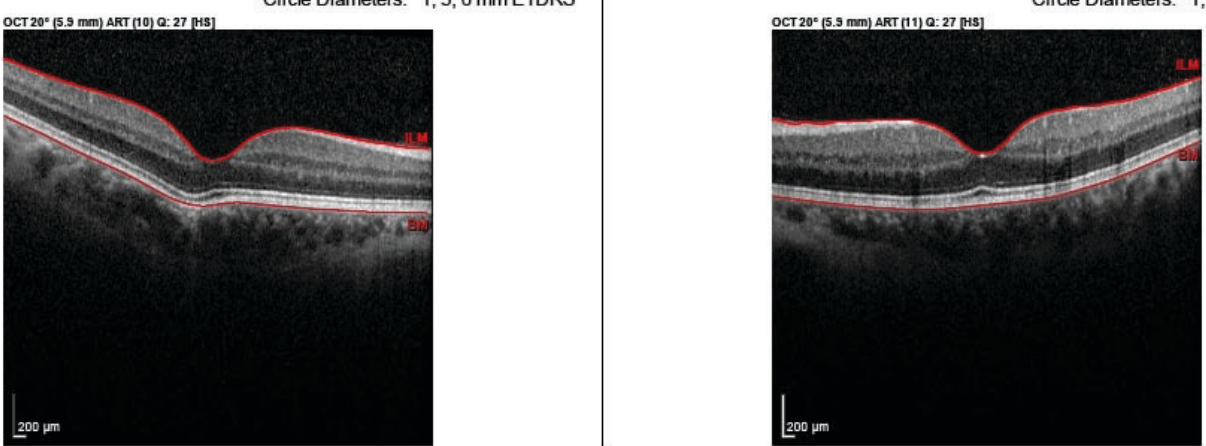
Figure 4: OCTs of the optic nerve heads with optic nerve swelling OS

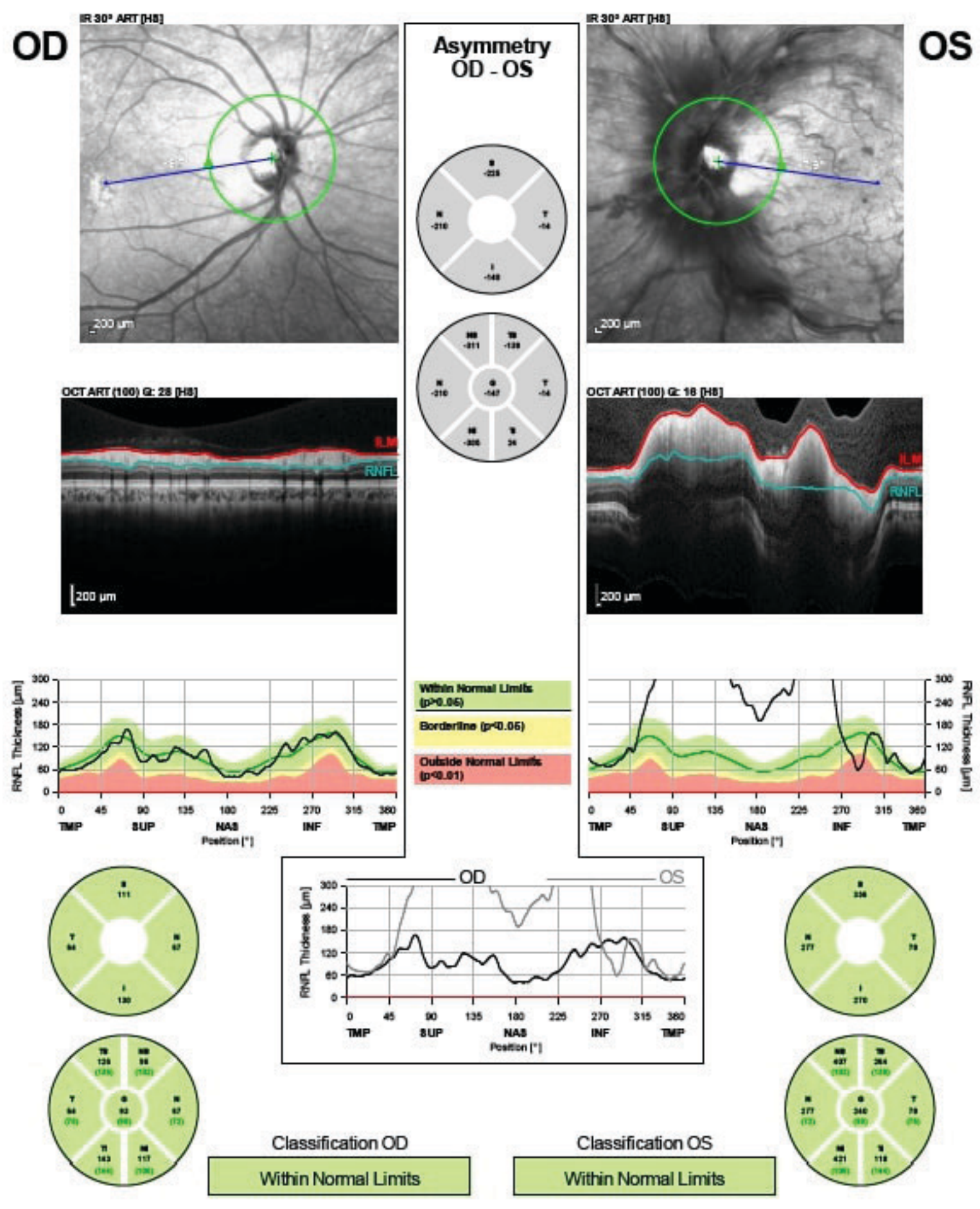




\section{Diagnosis and Treatment}

Given the recent history of trauma and the exam findings, the patient was diagnosed with a traumatic CRVO with a mild traumatic iritis OS. The case was reviewed with a retinal specialist who agreed on the diagnosis and etiology, pending lab results to rule out systemic blood dyscrasia. The patient was educated on the condition's self-resolving nature and was advised to avoid strenuous activities. He was prescribed prednisolone acetate three times a day os for the iritis and was scheduled to return in two weeks for an IOP check, gonioscopy, DFE, OCT Macula and OCT RNFL.

\section{Follow-Up and Additional Testing}

The patient was followed up two weeks after his initial exam, at which time resolution of the anterior chamber reaction was observed. The topical steroid was therefore tapered and the patient was monitored at monthly intervals for the development of neovascularization or worsening of clinical signs or symptoms. Over the course of his monthly follow-up visits, the patient's BCVA remained 20/20 OD, OS and he subjectively reported improvement and resolution in symptoms of peripheral blur OS. Gonioscopy was performed at all follow-up visits and no angle recession or neovascularization was noted. The patient demonstrated gradual resolution of the fundus findings and optic disc swelling at every follow-up, with significant improvement five months after the initial presentation (Figures 5, 6). Additional laboratory testing ruled out other etiologies that could account for CRVO in a young patient (Table 1).

Figure 5: Left posterior pole with resolution of retinal hemorrhages

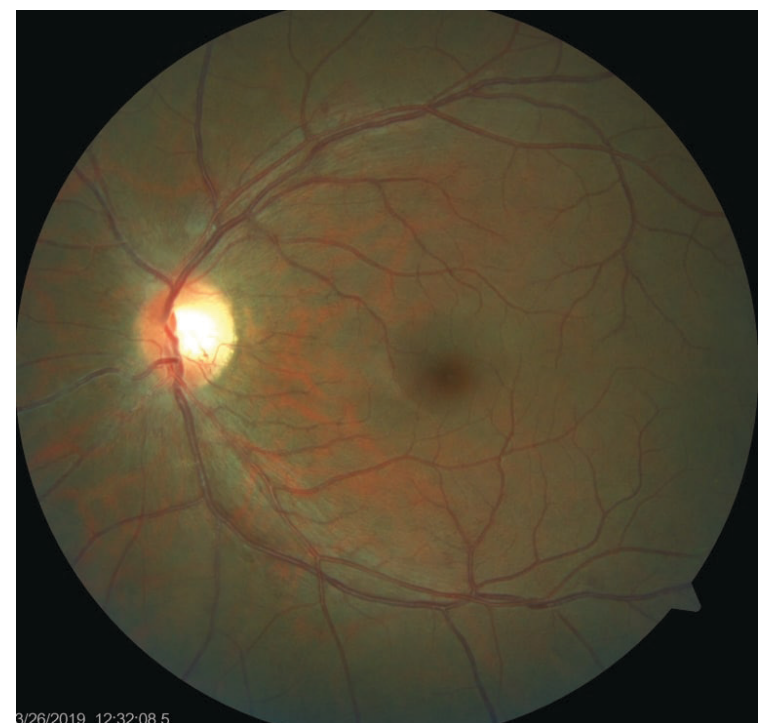

\section{DISCUSSION}

In the United States, traumatic ocular injuries are responsible for approximately 30,000 visits to the emergency department annually. ${ }^{3}$ Common traumatic ocular findings include contusion, ecchymosis, hyphema, cataract, glaucoma, vitreous hemorrhage, retinal hemorrhage and commotio retinae. Trauma-related CRVO highlighted in this case report is a very rare manifestation of ocular trauma and only a few examples have been described in the literature.

Clinical signs of CRVO include superficial and deep retinal hemorrhages in all four retinal quadrants, vessel engorgement and tortuosity, optic disc swelling, cotton wool spots, and cystoid macular edema. ${ }^{1}$ CRVOs typically occur in individuals over the age of 50 with risk factors that include hypertension, vascular disease, and African-American racial background. In addition, patients with end-organ damage due to diabetes mellitus are at an increased risk of CRVO compared to patients with uncomplicated diabetes mellitus. ${ }^{4}$ Other less common causes include oral contraceptive pills, collagen vascular disease, AIDS, protein S deficiency, protein C deficiency, antithrombin III deficiency, factor XII deficiency, antiphospholipid antibody syndrome and activated protein C resistance. 
Figure 6: OCT of left optic nerve head with resolution of optic nerve swelling
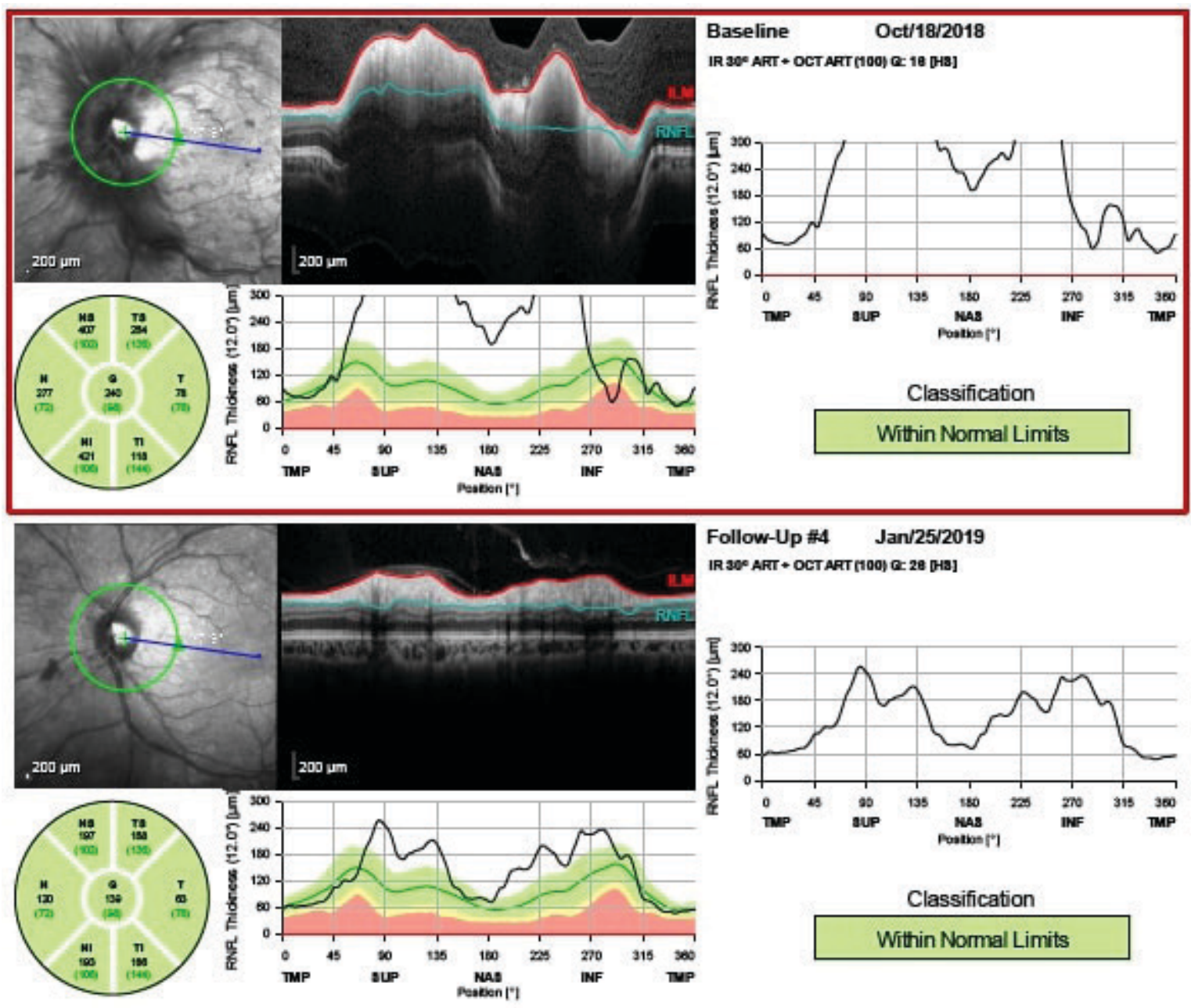

Follow-Up \#4 Jan/25/2019

IR 300 ART + OCT ART [100) \&: 28 [H:]
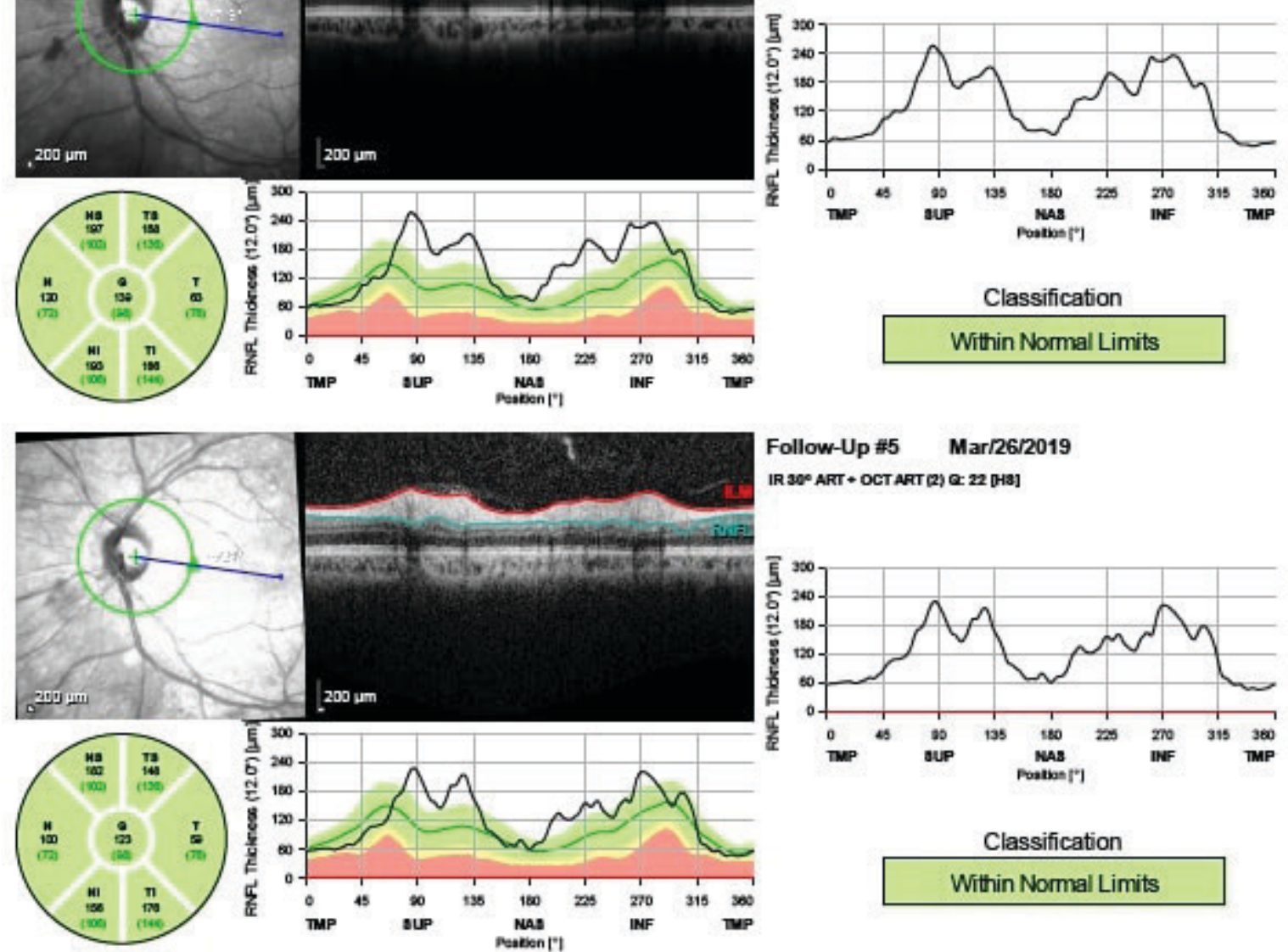

Follow-Up \#5 Mar/26/2019

IR 300 ART + OCT ART [2) \&: 22 [Hझ]
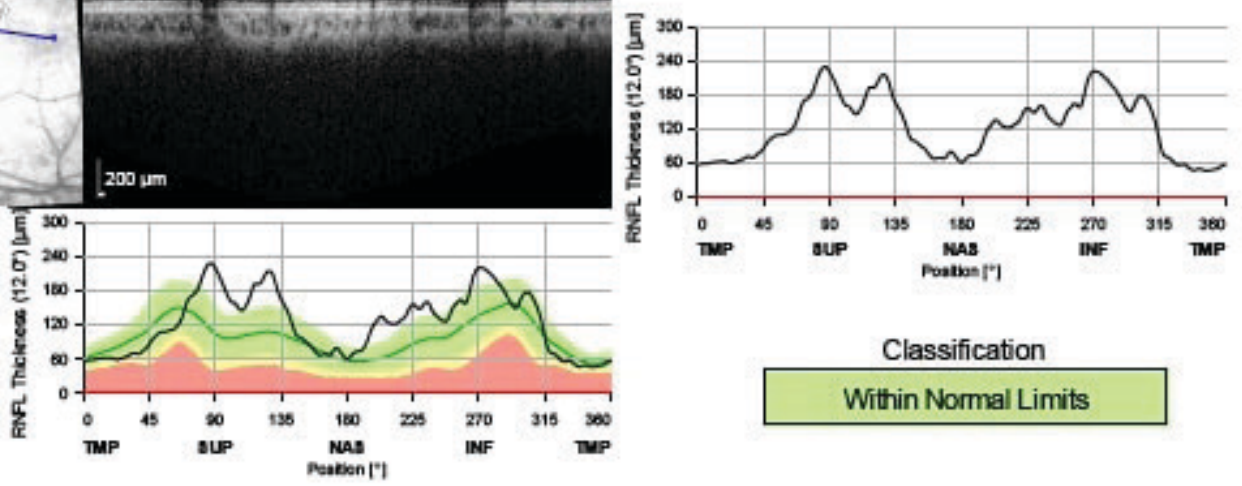

Classification

Within Normal Limits 
Table 1: Lab tests indicating no underlying etiologies to account for a CRVO in a young patient.

\begin{tabular}{|c|c|c|}
\hline Test & Result & Reference Range \\
\hline ESR & $4 \mathrm{~mm} / \mathrm{h}$ & $0-22 \mathrm{~mm} / \mathrm{h}$ \\
\hline Homocysteine & $9.8 \mu \mathrm{mol} / \mathrm{L}$ & $0.0-15.0 \mu \mathrm{mol} / \mathrm{L}$ \\
\hline ANA & Positive* & $<1: 40$ \\
\hline RPR & Non-reactive & Non-reactive \\
\hline MHA-TP & Non-reactive & Non-reactive \\
\hline LDL Cholesterol & $145.2 \mathrm{mg} / \mathrm{dL}$ & $0-130 \mathrm{mg} / \mathrm{dL}$ \\
\hline Total Cholesterol & $225 \mathrm{mg} / \mathrm{dL}$ & $0-200 \mathrm{mg} / \mathrm{dL}$ \\
\hline HDL & $59 \mathrm{mg} / \mathrm{dL}$ & $29-72 \mathrm{mg} / \mathrm{dL}$ \\
\hline Triglyceride & $102 \mathrm{mg} / \mathrm{dL}$ & $10-170 \mathrm{mg} / \mathrm{dL}$ \\
\hline WBC & $5.210^{3} / \mu \mathrm{L}$ & $3.5-10.010^{3} / \mu \mathrm{L}$ \\
\hline $\mathrm{RBC}$ & $5.410^{6} / \mu \mathrm{L}$ & $4.0-5.910^{6} / \mu \mathrm{L}$ \\
\hline Hemoglobin & $15.2 \mathrm{~g} / \mathrm{dL}$ & $12.0-18.0 \mathrm{~g} / \mathrm{dL}$ \\
\hline $\mathrm{HCT}$ & $46.0 \%$ & $36.0-52 \%$ \\
\hline $\mathrm{MCV}$ & $85.9 \mathrm{fL}$ & $81-100 \mathrm{fL}$ \\
\hline $\mathrm{MCH}$ & $28.3 \mathrm{pg}$ & $27-35 \mathrm{pg}$ \\
\hline MCHC & $33.0 \mathrm{~g} / \mathrm{dL}$ & $33.0-36.0 \mathrm{~g} / \mathrm{dL}$ \\
\hline RDW & $13.0 \%$ & $12.3-16.4 \%$ \\
\hline Platelet Count & $22210^{3} / \mu \mathrm{L}$ & $150-45010^{3} / \mu \mathrm{L}$ \\
\hline MPV & $9.1 \mathrm{fL}$ & 7.4-10.4 fL \\
\hline Neutrophil \% & $61.5 \%$ & $45.0-75.0 \%$ \\
\hline Lymphocyte \% & $28.0 \%$ & $18.0-43.0 \%$ \\
\hline Monocyte \% & $7.3 \%$ & $2.0-14.0 \%$ \\
\hline Eosinophil \% & $2.6 \%$ & $0.0-8.0 \%$ \\
\hline Basophil \% & $0.6 \%$ & $0.0-2.0 \%$ \\
\hline Neutrophil (\#) & $3.2010^{3} / \mu \mathrm{L}$ & $1.6-7.510^{3} / \mu \mathrm{L}$ \\
\hline Lymph (\#) & $1.5010^{3} / \mu \mathrm{L}$ & $0.6-4.310^{3} / \mu \mathrm{L}$ \\
\hline Mono (\#) & $0.4010^{3} / \mu \mathrm{L}$ & $0.1-1.410^{3} / \mu \mathrm{L}$ \\
\hline $\operatorname{Eos}(\#)$ & $0.1010^{3} / \mu \mathrm{L}$ & $0.0-0.810^{3} / \mu \mathrm{L}$ \\
\hline Baso (\#) & $0.0010^{3} / \mu \mathrm{L}$ & $0.0-0.210^{3} / \mu \mathrm{L}$ \\
\hline PTime & 13.1 seconds & $12.0-14.7$ seconds \\
\hline PTT & 33.4 seconds & 23.6-36.6 seconds \\
\hline Anticardiolipin AB, IgG & $<9 \mathrm{GPL} \mathrm{U} / \mathrm{mL}$ & 0-14 GPL U/mL \\
\hline Anticardiolipin AB, IgM & < $9 \mathrm{MPL} \mathrm{U} / \mathrm{mL}$ & 0-12 MPL U/mL \\
\hline
\end{tabular}

* Positive ANA at a concentration of 1:40, at which some subjects in the normal population test positive without systemic issues. ANA was not found to be positive at more dilute concentrations. 
CRVO is sub-divided into non-ischemic and ischemic. An ischemic CRVO is defined as capillary non-perfusion on fluorescein angiogram (FA) of more than 10 disc diameters. ${ }^{2}$ In patients with ischemic CRVO, elevated levels of protein related to hypoxia, angiogenesis and inflammation make development of neovascularization more likely. ${ }^{5}$ The visual prognosis in CRVO can vary from 20/20 to hand motion. Vision loss due to extensive hemorrhaging at initial presentation can improve as the hemorrhages resolve, or vision can conversely worsen due to prolonged retinal non-perfusion. Non-perfusion can subsequently cause the increased production of vascular endothelial growth factor (VEGF), which may lead to macular edema and neovascularization. ${ }^{5}$ Our patient's CRVO was diagnosed as non-ischemic due to the lack of severe vision loss and the absence of a relative afferent pupillary defect (RAPD). A FA was not ordered due to the low degree of suspicion of ischemic etiology in this case.

Trauma-related CRVOs are rare in the literature. According to Hayreh et al., 10 out of 680 individuals had a CRVO after blunt trauma. ${ }^{6} \mathrm{El}$ Asri et al. reported the case of a 35-year-old male who sustained ocular trauma due to a blast injury in a conflict zone and presented with a sudden decline in vision due to a CRVO. This case differs from our patient's case since this patient experienced decreased vision and further analysis showed a positive factor II G20210A gene mutation. Our patient has not yet had genetic testing, and it is possible that he may have a gene mutation that predisposes him to the occurrence of CRVO. Alvarez and Noble reported a case of a combined central retinal artery occlusion (CRAO) and CRVO in a 28-year-old who was jabbed in the eye as opposed to the CRVO without arterial occlusion that occurred in our patient. ${ }^{8}$

In a typical case of CRVO, the pathogenesis is thought to involve obstruction of the central retinal venous outflow. Different factors such as vascular changes, hematologic and thrombotic tendencies, and local anatomical susceptibility can also contribute to the development of a CRVO. ${ }^{1}$ Our patient sustained blunt trauma to the eye during Jiu Jitsu, resulting in acute mechanical deformation of the eye and subsequent collapse of the central retinal vein against the lamina cribrosa. Alternatively, increased IOP can create a greater pressure-difference as the vein exits the eye. However, this etiology was ruled out because our patient had normal, symmetric IOPs, absence of a hyphema and no angle recession on gonioscopy. Finally, congenital anomalies, such as increased vascular tortuosity or anomalous anatomic configuration, may affect the hemodynamics of venous outflow and predispose the central retinal vein to thrombosis. ${ }^{9}$ Our patient had an acute CRVO with likely gradual development of optic nerve head edema. This may be why his symptoms of peripheral visual blur were not acute and presented after one week.

The natural course of a traumatic CRVO in young adults is spontaneous resolution over three to six months, with a minimal decrease in best corrected visual acuity. Fong and Schatz reviewed 488 cases and reported that these patients typically recover in an average of 12.2 weeks. ${ }^{10}$ With regard to treatment, the Study of Comparative Treatments for Retinal Vein Occlusion 2 (SCORE2) established that all three anti-VEGF agents (aflibercept, bevacizumab and ranibizumab) are effective in the treatment of macular edema. ${ }^{11}$ The prognosis of CRVO usually depends on whether it is non-ischemic or ischemic. Non-ischemic CRVO typically has a better final visual outcome.

\section{CONCLUSION}

Though CRVOs typically occur in the vasculopathic population aged 50 years and older, this case report describes the unusual sequelae of a CRVO in a young patient following blunt trauma. As eye care providers, it is important that we are aware of common complications as well as more rare manifestations of ocular trauma. A thorough case history and appropriate, prompt management should help to prevent further ocular complications. •

\section{CORRESPONDENCE}

Ailia Ahmed, OD, Email: ailia72@gmail.com

\section{REFERENCES}

1. McAllister IL. Central retinal vein occlusion: a review. Clin Exp Ophthalmol 2012;40:48-58.

2. Friedman NJ, Kaiser PK, Pineda R. The Massachusetts Eye and Ear Infirmary Illustrated Manual of Ophthalmology. China: Elsevier, 2014.

3. Canner JK, Haring RS, Schneider EB, et al. Epidemiology of sports-related eye injuries in the United States. JAMA Ophthalmol 2016;134(12):1382-90.

4. Comer GM, Stein JD, Stem MS, et al. A longitudinal analysis of risk factors associated with central retinal vein occlusion. Ophthalmology 2013;120:362-70.

5. Agostini HT, Ehlken C, Feltgen N, et al. Increased expression of angiogenic and inflammatory proteins in the vitreous of patients with ischemic central retinal vein occlusion. PLoS ONE 2015;10(5):1-15.

6. Hayreh SS, Podhajsky PA, Zimmerman MB. Natural history of visual outcome in central retinal vein occlusion. Ophthalmology 2011;118:119-33.
7. El Asri F, Fenolland JR, Giraud JM, et al. [A rare trauma-associated cause of central retinal vein occlusion in a young subject.] $\mathrm{J} \mathrm{Fr}$ Ophtalmol 2012;35(6):426-31. (in French)

8. Alvarez EV, Noble MJ. Combined occlusion of the central retinal artery and central retinal vein following blunt ocular trauma: a case report. Br J Opthalmol 1987;71:834-6.

9. Cankaya C, Cumurcu T, Demirel S, et al. Traumatic optic neuropathy and central retinal artery occlusion following blunt ocular trauma. J Clin Med Res 2011;3(1):55-7.

10. Fong ACO, Schatz H. Central retinal vein occlusion in young adults. Surv Ophthalmol 1993;37:393-417.

11. Bressler NM. Treatment of macular edema due to central retinal vein occlusion: another score for repackaged bevacizimab. JAMA 2017 May 23;317(20):2067-9. 